\title{
Potential prognostic value of miR-132 and miR-212 expression in MCRPC patients
}

\author{
Mariano Pontico ${ }^{1}$, Viviana Frantellizzi ${ }^{2}$, Luca Cindolo ${ }^{3}$, Giuseppe De Vincentis ${ }^{2}$ \\ ${ }^{1}$ Program in Morphogenesis \& Tissue Engineering, Department of Medico-Surgical Sciences and Biotechnologies, \\ Sapienza University of Rome, Rome, Italy; \\ ${ }^{2}$ Department of Radiological Sciences, Oncology and Anatomical Pathology, Sapienza, "Sapienza" University of Rome, Rome, Italy; \\ ${ }^{3}$ Department of Urology, "Villa Stuart" Private Hospital, Rome, Italy.
}

Submitted 13 November 2020; Accepted 11 January 2021

To the Editor,

we have been very pleased to read the interesting work proposed by Salemi et al. (1) regarding the expression of specific fragments of microRNA (miRNA), particularly miR-132 and miR-212, as potential key regulators in prostate cancer (PCa). As outlined by the Authors, the altered expression of miRNAs in cancer pathogenesis represents a well-consolidated knowledge in the current literature $(2,3)$. More specifically, both miR-212 and miR-132 regulate subsets of genes involved in tumor progression in several tumor cell types as PCa, proving a central role in tumorigenesis, cell adhesion, and angiogenesis. In addition, a strong association between miR-132 expression and high Gleason score PCa has been lately depicted. Several studies have identified specific miRNA expression profiles in the serum of cancer patients, as well as an increased expression of certain miRNAs in the blood of patients with metastatic PCa compared with non-metastatic $\mathrm{PCa}$ (4). Thus, miRNA expression profiles could act as potential biomarkers for PCa diagnosis and prognosis.

In this context, data achieved by Salemi et al. remarked an increased expression of miR-132 and miR-212 in PCa tissue compared to control and, by contrast, a reduced expression of miR132 and miR-212 in metastatic lymph node. The intriguing findings obtained by this case are likely to suggest a different biologic behavior between the primary PC and metastatic lymph nodes. Recently, we described the outcomes obtained in a real-world setting by the biggest cohort of mCRPC patients undergoing 223-Ra radiometabolic treatment in our country and one of the most extensive in the whole of Europe (5).

The risk of developing metastatic localizations during follow-up ranges from 26\% to 38\% after primary radical approaches and about $4 \%$ of the patients are ex-Novo diagnosed with metastatic disease. Bone-metastatic PCa occurs in up to $90 \%$ of mCRPC patients and represents a leading cause of morbidity in these subjects, being associated with significant clinical complications and quality of life impairment. Although there is no consensus regarding the very optimal treatment of high-risk or locally advanced PCa, in the last years, multi-modal therapy has demonstrated a clinical advantage and many clinical trials are ongoing to evaluate the best clinical therapeutic approach for each patient. Thus, there is an urgent need to better understand high-risk PCa prognosis using new biomarkers (i.e., histological and molecular genetics patterns), aiming to reach the most appropriate optimization of current treatment strategies to improve outcomes, avoid or postpone complications, and accordingly to enhance the patient's overall quality of life.

Currently, it is still unequivocal how to best apply them in a real clinical practice setting, particularly in case of decisions concerning treatment sequencing and combination options (6).

In general, for mCRPC patients, androgen deprivation therapy with or without chemotherapy was recommended by EAU guidelines $(7,8)$. With the successful application of cytoreductive surgery for metastatic cancers and the signs of progress achieved in surgical and radiotherapy techniques, the role of the cytoreductive prostatectomy approach for mCRPC has gained great interest. As pointed out by the results of our study (9), cytoreductive prostatectomy may have the potential to enhance mCRPC disease control, however, the lack of randomized controlled trials and the low level of evidence in the current literature preclude any firm conclusions on the benefit of cytoreductive strategy in MCRPC and to clearly identify the patients who would benefit most from their primary PCa ablation.

Based on this growing experience and believing in the great potential of the integration of the perspective emerged from molecular findings by Salemi et al. with our PCa patients cohort, we focus on the opportunity to cross-reference the miR expression analysis in $\mathrm{mCRPC}$ enrolled for 223-Ra treatment, to evaluate the clinical and prognostic relevance of miR aberrations in this peculiar group of PCa patients. More in detail, different groups of patients clustered for multiple PCa biological (Gleason Score, histopathologic and molecular patterns), biochemical (PSA, LDH, tALP) (10), and clinical features (TNM score, performance status, secondary skeletal burden) could be designed, aiming to assess the precise relation, if any, between miR expression with every single variable.

The final goal would consist of the achievement of the most effective treatment sequence possible, relying on the individual

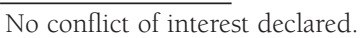


clinical and biomolecular characteristics specific of each patient, in a real patient-centered treatment approach (11). In this direction, it would be very interesting other than useful to assess molecular miRNAs patterns of PCa bone secondary localizations, investigating whether these peculiar pathological entities act like the lymph nodal ones rather than the primary PCa discussed in the case reported by Salemi et al., thus respectively showing under- or over-expression of miR132 and miR-212.

A detailed analytical assessment of these new molecular variables in MCRPC patients could eventually lead to a clinically useful prognostic stratification of which kind of PCa patient would rather benefit from a specific treatment strategy, such as 223-Ra treatment.

The outcomes of molecular miRNAs expression assessment in skeletal PCa metastases could potentially help to shed light on the role of primary tumor cytoreduction in MCRPC and its still enigmatic biological causes. Several studies are suggesting the existence of a critical interaction between primary tumors, their circulating and disseminated cells, and the development and maintenance of secondary lesions, via a complex connecting network. This tangled connection could justify how primary tumor ablation is demonstrated in many cases to prevent the development of new metastases and even promote their regression in a phenomenon known as the abscopal effect $(12,13)$.

Even if the biological mechanisms underlying this hypothesis are not yet known in detail, most of the actual evidence confirm that ablative treatment of the primitive tumor, purposing to reduce the local load of disease, is able to positively influence the biological behavior of secondary locations too, along with their response to systemic therapies. Whether these theories apply to all or only specific solid tumors remains still to be determined.

In this regard, we hope in the development of further detailed studies with a larger number of PCa patients that could definitely lead to many solid achievements, with the purpose to help the determination of peculiar miRNA expression profiles for $\mathrm{PCa}$, supporting its early diagnosis, stratification, and prognosis clinical workup.

\section{REFERENCES}

1. Salemi M, Pettinato A, Fraggetta F, et al. Expression of miR-132 and miR-212 in prostate cancer and metastatic lymph node: Case report and revision of the literature. Arch Ital Urol Androl. 2020; 92:209-210.

2. Hassan O, Ahmad A, Sethi S, and Sarkar FH. Recent updates on the role of microRNAs in prostate cancer. J Hematol Oncol. 2012; 5:9.

3. Zhang W, Edwards A, Fan W, et al. miRNA-mRNA correlation-network modules in human prostate cancer and the differences between primary and metastatic tumor subtypes. PLoS One. 2012; 7:e40130.

4. Chen ZH, Zhang GL, Li HR, et al. A panel of five circulating microRNAs as potential biomarkers for prostate cancer. Prostate. 2012; 72:144352 .

5. Frantellizzi V, Monari F, Mascia M, et al. Radium-223 in mCPRC patients: a large real-life Italian multicenter study. Minerva Urol Nefrol 2020. doi: 10.23736/S0393-2249.20.03808-4. Epub ahead of print.

6. Frantellizzi V, Lazri J, Pontico M, et al. Bone pain palliation outcomes and possibility of Radium-223 re-treatment in $m C R P C$. Arch Ital Urol Androl. 2020; 92:196-199

7. De Vincentis G, Follacchio GA, Frantellizzi V, et al. 223Ra-dichloride therapy in an elderly bone metastatic castration-resistant prostate cancer patient: a case report presentation and comparison with existing literature. Aging Clinical and Experimental Research. 2017; 30:677-80.

8. Ricci M, Frantellizzi V, Bulzonetti N, De Vincentis G. Reversibility of castration resistance status after Radium-223 dichloride treatment: clinical evidence and review of the literature. Int J Radiat Biol. 2019; 95:554-561.

9. Frantellizzi V, Costa R, Mascia M, et al. Primary radical prostatectomy or ablative radiotherapy as protective factors for patients with $m C R P C$ treated with radium-223 dichloride: an Italian multicenter study. Clin Genitourin Cancer. 2020; 18:185-91.

10. De Vincentis G, Follacchio GA, Frantellizzi V, et al. Prostate-specific antigen flare phenomenon during 223Ra-dichloride treatment for bone metastatic castration-resistant prostate cancer: a case report. Clinical Genitourinary Cancer. 2016; 14:e529-e33.

11. Prelaj A, Rebuzzi SE, Buzzacchino F, et al. Radium-223 in patients with metastatic castration-resistant prostate cancer: Efficacy and safety in clinical practice. Oncol Lett. 2019; 17:1467-76.

12. Abuodeh Y, Venkat P, Kim S. Systematic review of case reports on the abscopal effect. Curr Probl Cancer. 2016; 40:25-37.

13. Yilmaz MT, Elmali A, Yazici G. Abscopal Effect, From Myth to Reality: From Radiation Oncologists' Perspective. Cureus. 2019;11:e3860.

\section{Correspondence}

Mariano Pontico, MD

mariano.pontico@uniromal.it

Program in Morphogenesis \& Tissue Engineering, Department of Medico-Surgical Sciences and Biotechnologies, Sapienza University of Rome, Rome (Italy)

Viviana Frantellizzi, MD, PhD (Corresponding Author) viviana.frantellizzi@uniromal.it

Department of Radiological Sciences, Oncology and Anatomical
Pathology, Sapienza, "Sapienza" University of Rome Viale Regina Elena 324, 00161 Rome (Italy)

Luca Cindolo, MD, PhD

lucacindolo@virgilio.it

Department of Urology, "Villa Stuart" Private Hospital, Rome (Italy)

Giuseppe De Vincentis, MD, PhD

giuseppe.devincentis@uniromal.it

Department of Radiological Sciences, Oncology and Anatomical

Pathology, Sapienza, "Sapienza" University of Rome, Rome (Italy) 